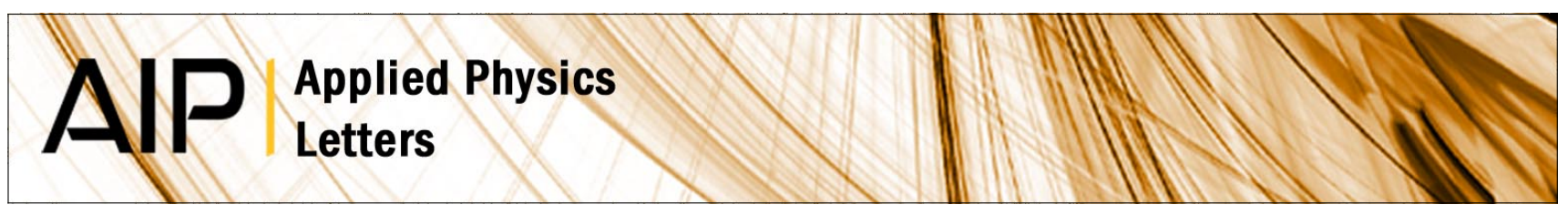

\title{
Aharonov-Bohm effect induced by light in a fiber
}

Igor O. Kulik and Alexander S. Shumovsky

Citation: Appl. Phys. Lett. 69, 2779 (1996); doi: 10.1063/1.117673

View online: http://dx.doi.org/10.1063/1.117673

View Table of Contents: http://apl.aip.org/resource/1/APPLAB/v69/i18

Published by the American Institute of Physics.

Additional information on Appl. Phys. Lett.

Journal Homepage: http://apl.aip.org/

Journal Information: http://apl.aip.org/about/about_the_journal

Top downloads: http://apl.aip.org/features/most_downloaded

Information for Authors: http://apl.aip.org/authors

\section{ADVERTISEMENT}

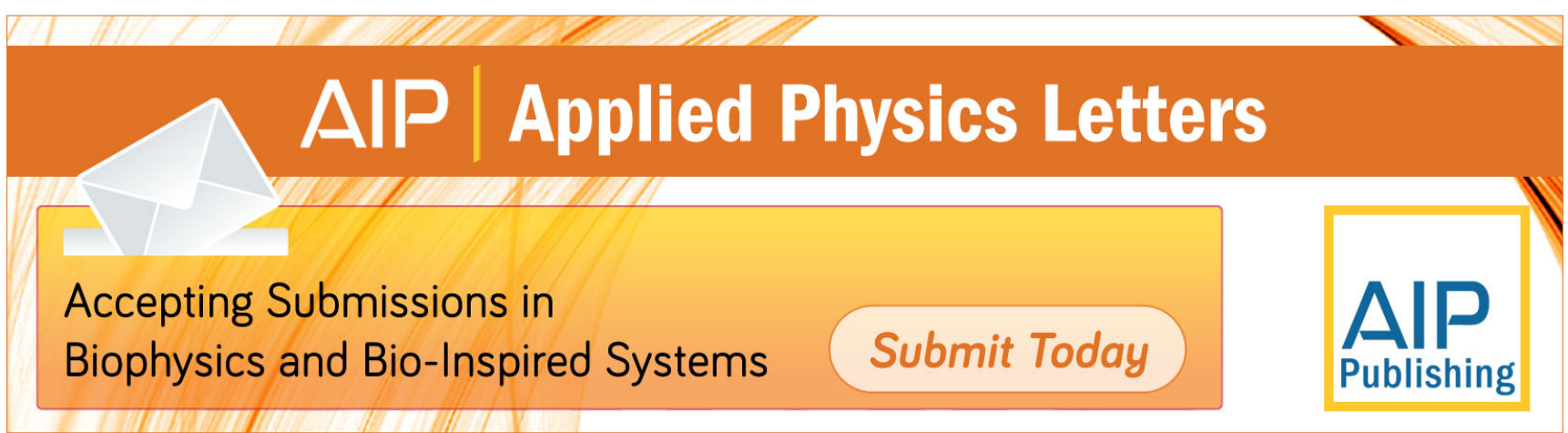




\title{
Aharonov-Bohm effect induced by light in a fiber
}

\author{
Igor O. Kulik ${ }^{\mathrm{a})}$ and Alexander S. Shumovsky ${ }^{\mathrm{b})}$ \\ Department of Physics, Bilkent University, Bilkent 06533, Ankara, Turkey
}

(Received 1 March 1996; accepted for publication 2 August 1996)

\begin{abstract}
A weakly coupled normal-metal ring surrounding an optical fiber is considered under the condition that the frequency of light in fiber is larger than the conduction bandwidth of the metal. It is shown that in the presence of static magnetic field parallel to the fiber axis, the resistance of the ring is a nonmonotone function of the optical intensity and an oscillating function of the static magnetic flux with period equal to flux quantum $h c / e$. The temperature dependence of oscillations requires that inelastic mean free path of electrons is larger than the ring size, and does not relate to the energy level spacing to temperature ratio. (C) 1996 American Institute of Physics. [S0003-6951(96)01342-3]
\end{abstract}

It has been recognized that quantum effects in mesoscopic structures related to the vector potential of the electromagnetic field may lead to high-precision and highsensitivity measurements at low temperatures (e.g., see Ref. 1). So far the quantum interferometry of normal metallic loops was applied to static or slowly time-varying magnetic fields. The objective of this letter is to consider the highfrequency mesoscopic effects induced by light in an optical fiber piercing the metallic loop and thereby to demonstrate the possibility of nondemolition control of light propagation through the fiber.

It is well known that the Aharonov-Bohm effect ${ }^{2}$ at dc excitation manifests itself in the appearance of a persistent current in a metallic loop periodic as a function of magnetic flux with the period of flux quantum $h c / e^{3,4}$ and in the resistance oscillations in the loop incorporated into an external circuit $^{5}$ with the same period. The first type of the experiment was carried out by Chandrasekhar et al. ${ }^{6}$ and by Mally et $a .^{7}{ }^{7}$ Resistance oscillations have been observed in Ref. 1 and references therein.

An important case of an ac field of high frequencies $\omega \gg v_{F} / R$ where $v_{F}$ is the Fermi velocity and $R$ is the ring radius has been considered by Aronov et al ${ }^{8,9}$ under the assumption that the space dependent time-varying electromagnetic field produces the static electron energy minibands in the ring. The minibands have been suggested ${ }^{6,9}$ to appear due to electron motion in a time-averaged electrostatic potential periodic with coordinate along ring circumference, produced by the square of an ac electric field. ${ }^{10}$ However, in the quantum case, an electron reflection from an oscillating potential causes a time-dependent phase shifts resulting in an effective chaotization of the phase of electron wave function, except at energy multiples of $\hbar \omega$.

In this letter we consider the case of much higher (optical) frequency $\omega>\Delta E / \hbar$ where $\Delta E$ is the width of the electron conduction band of the metal. Under this condition, the inelastic scattering of electrons is prohibited if the separation between the conduction band and higher nonoccupied bands

\footnotetext{
a) Also at B. Verkin Institute for Low Temperature Physics and Engineering, Academy of Science of Ukraine, Khar'kov, Ukraine.

b) Also at Bogolubov Laboratory of Theoretical Physics, Joint Institute for Nuclear Research, Dubna, Russia. Electronic mail: shumo@fen.bilkent.edu.tr
}

of a metal is larger than $\hbar \omega$. In this case, the magnetic component of an electromagnetic field represents the main source of the electron wave function phase shift. The effect of oscillating magnetic field results in the modulation of the electron transmission amplitude between the parts of the ring. Due to the quantum interference of electron waves in an oscillating potential, the dependence of the loop resistance on the ac field amplitude becomes a nonmonotone character.

An example of the high-frequency Aharonov-Bohm effect is provided by a small metallic ring surrounding an optical fiber [Fig. 1(a)]. We show here that the resistance of the ring has a nonmonotone dependence on the ac power and oscillates as a function of static magnetic field applied parallel to optical fiber axis. We assume that the ring is inhomogeneous and that the ac electric field is concentrated near the narrowings $A, B, \ldots$. Hopping of electrons near these points will be influenced by a phase factor emerging from the vector potential $\mathbf{A}(\mathbf{r}, t)$ of the ac field.

Consider for simplicity a one-dimensional loop in the tight-binding approximation with two transmittance amplitudes $t_{1}, t_{2}$ at points $A, B$, connecting two parts of the ring at $n=n^{\prime}=n_{1}$ and $n=n^{\prime}=n_{2}$, much smaller than the hopping amplitude $t_{0}$ between the nearest points inside upper and lower parts of the ring [Fig. 1(c)]. Here $n$ enumerates the sites along the ring. A weakly coupled loop of equal length chains $\left(N=n_{2}-n_{1}\right)$ is formed along the contour $A A^{\prime} B^{\prime} A$. This model can be solved exactly and we hope that the result will remain qualitatively valid at $t_{1,2}-t_{0}$. Further simplification, in the spirit of that philosophy, consists in the passage to the configuration depicted by the Fig. 1(d) with two parallel weakly coupled infinite chains $(\ldots A \ldots B \ldots)$ and $\left(\ldots A^{\prime} \ldots B^{\prime} \ldots\right)$ connected at the lower part to the thermal reservoirs $R_{1}$ and $R_{2}$, holding at different static voltages $V_{1}$ and $V_{2}$.

The system in question is described by a model Hamiltonian

$$
\begin{aligned}
& H=-t_{0} \sum_{n}\left(a_{n}^{+} a_{n+1}+b_{n}^{+} b_{n+1}\right)+h . c .+H_{i n t}, \\
& H_{\text {int }}=-t_{1} a_{n_{1}}^{+} b_{n_{1}} e^{i \alpha_{1}}-t_{2} a_{n_{2}}^{+} b_{n_{2}} e^{i \alpha_{2}}+h . c .
\end{aligned}
$$

where $a_{n}, b_{n}$ are the electron annihilation operators. The phases of transmission amplitudes at the contraction points $n_{1}, n_{2}$ are 


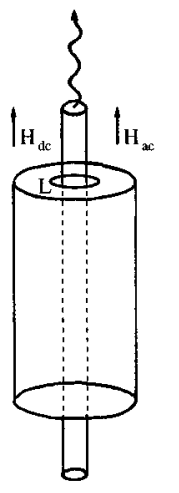

(a)

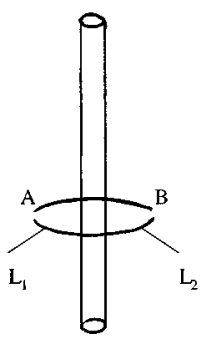

(b)

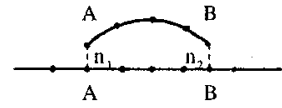

(c)

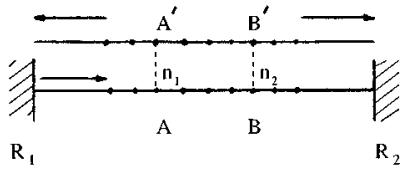

(d)

FIG. 1. (a) Mesoscopic metallic loop $L$ surrounding the core of an optical fiber. The ac magnetic field $\mathbf{H}_{\mathrm{ac}}$ of the $T E_{01}$ mode lies along the $z$-axis as well as the static field $\mathbf{H}_{\mathrm{dc}}$. (b),(c) Scheme of $1 d$ loop with the external leads $L_{1}, L_{2}$ weakly coupled at points $A, B$. (d) Model of an ac normal-metal interferometer adopted in this letter. $R_{1}, R_{2}$ are the thermal reservoirs held at voltages $\mp V / 2$, respectively.

$$
\alpha_{i}=\alpha_{i}^{0}+A_{i} \sin \left(\omega t+\delta_{i}\right)
$$

where $\alpha_{i}^{0}$ accounts for the effect of a dc magnetic field applied perpendicular to the plane of the ring

$$
\alpha_{1}^{0}-\alpha_{2}^{0}=\frac{2 \pi \Phi_{\mathrm{dc}}}{\Phi_{0}}, \quad \Phi_{\mathrm{dc}}=\int \mathbf{B} d \mathbf{S}, \quad \Phi_{0}=\frac{h c}{e}
$$

while $A_{i}$ are the amplitudes of high-frequency field at corresponding points. Hamiltonian (1) is Fourier-transformed into the following:

$$
\begin{aligned}
& H_{i n t}=\sum_{n=-\infty}^{\infty} H_{i n t}^{(n)} e^{i n \omega t}, \\
& H_{i n t}^{(n)}=-\sum_{j=1}^{2} t_{j} e^{i \alpha_{j}^{0} J_{n}\left(A_{j}\right) a_{n_{j}}^{+} b_{n_{j}},}
\end{aligned}
$$

where $J_{n}(z)$ is the Bessel function. It is not necessary to take into account the contribution of $H_{i n t}^{(n)}$ at $n \neq 0$ because the scattering events are forbidden under the condition $4 t_{0}<\hbar \omega$. In fact, they lead to the change of energy $\varepsilon_{k}=\varepsilon_{k^{\prime}}$ $+n \hbar \omega$. Within the framework of the one-dimensional hopping model, there is no other electronic band except a single one with the width $4 t_{0}$. The model can be applied to a real metallic system if $\hbar \omega$ is less than the separation between the conduction band and higher non-occupied bands.

By perturbation, the forward $(+)$ and backward (-) scattering probabilities between the plane-wave states

$$
\psi_{k}=\sum_{n} e^{i k n} a_{n}^{+}|0\rangle, \quad \phi_{k}=\sum_{n} e^{i k n} b_{n}^{+}|0\rangle
$$

are $W_{ \pm k}$ where

$$
\begin{aligned}
& W_{k}[A+B \cos (\alpha+2 k L)] /\left|v_{k}\right|, \\
& A=\sum_{j=1}^{2}\left[t_{j} J_{0}\left(A_{j}\right)\right]^{2}, \\
& B=2 \sum_{j=1}^{2} t_{j} J_{0}\left(A_{j}\right) .
\end{aligned}
$$

Here $L$ is the total length of the loop $A B B^{\prime} A^{\prime}$, the phase $\alpha=2 \pi \Phi_{d c} / \Phi_{0}, v_{k}=\partial \varepsilon_{k} / \partial k$ is the electron group ve- locity, and $\varepsilon_{k}=-2 t_{0} \cos k$. In the steady state, the populations $f_{ \pm k}$ and $f_{ \pm k}^{\prime}$ of electron states can be obtained from the kinetic equation. After that, it should be Eq. (7) which is now written as follows:

$$
\begin{aligned}
f_{ \pm k}^{\prime}[ & {\left[W_{0}\left(1-f_{ \pm k}\right)+W_{ \pm k}\left(1-f_{\mp k}\right)\right] } \\
= & \left(1-f_{ \pm k}^{\prime}\right)\left[W_{0} f_{ \pm k}+W_{ \pm k} f_{\mp k}\right] .
\end{aligned}
$$

The electron distribution $f_{n}^{\prime}$ in the lower chain corresponds to the electrons emerging from the thermal reservoir $R_{1}$ (at $K>0)$ and therefore is equal to the equilibrium distribution function $f_{0}(\varepsilon-e V / 2)$ whereas $f_{-K}^{\prime}=f_{0}(\varepsilon+e V / 2)$ corresponds to the electrons emerging from $R_{2}$. The current $J$ in the lower chain is determined by the difference between the number of electrons moving to the right and to the left. Solving for $f_{1}^{\prime}, f_{2}^{\prime}$ from (7), we obtain

$$
\begin{aligned}
J= & \int_{0}^{\pi} \frac{d k}{2 \pi} W_{0}\left[\frac{W_{k}}{W_{0}+W_{k}}+\frac{W_{-k}}{W_{0}+W_{-k}}\right]\left[f_{0}\left(\varepsilon_{k}-\frac{e V}{2}\right)\right. \\
& \left.-f_{0}\left(\varepsilon_{k}+\frac{e V}{2}\right)\right] .
\end{aligned}
$$

The contribution to conductance $G=d J / d V$ due to the interchain scattering is

$$
\begin{aligned}
G= & \frac{e^{2}\left(t_{1}^{2}+t_{2}^{2}\right) W_{0}}{2 h T} \int_{0} \frac{\pi W_{0}\left[W_{k}+W_{-k}\right]+2 W_{k} W_{-k}}{\left[W_{0}+W_{k}\right]\left[W_{0}+W_{-k}\right]} \\
& \times \frac{d k}{\left|v_{k}\right| \cosh ^{2}\left[\left(\varepsilon_{k}-\mu\right) / 2 T\right]}
\end{aligned}
$$

We now note that Eq. (9) is equivalent to the Landauer formula for the conductance ${ }^{14,15}$ at transmission probability $|t|$. The logarithmic divergence in the integral (a) is removed if inelastic scattering and $3 d$ band effects are taken into account. At low temperature, the main contribution to integral comes from the vicinity of Fermi surface where these effects are insignificant.

The largest contribution to the conductance oscillations described by the above formulas corresponds to the mode $\mathrm{TE}_{01}$ of the fiber field (see Ref. 13). The typical magnitude of the conductance change in (10) is of the order of universal 


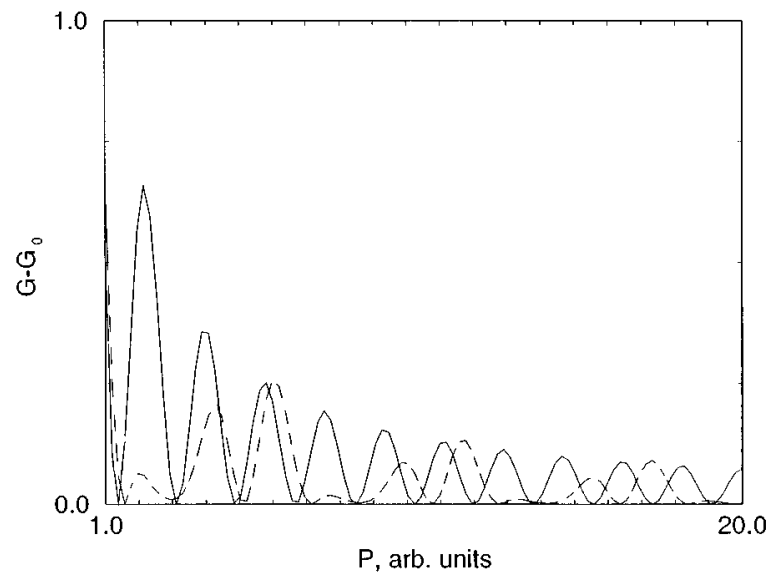

FIG. 2. dc conductance of the loop vs square root of ac power: solid line, $A_{1}: A_{2}=1: 1$; dotted line, $A_{1}: A_{2}=1: 2$. Change in the conductance is normalized with respect to static conductance oscillation amplitude.

conductance quantum $2 e^{2} / h \simeq 1 / 12.9 \mathrm{k} \Omega$. The size of the loop should be of the order of a few wavelengths of light to ensure that the total flux piercing the ring in the $\mathrm{TE}_{01}$ mode is not equal to zero.

It follows from Eq. (9) that the dependence of $G$ on phase $\alpha$ and on the electromagnetic field amplitude leads to two different effects. First, the oscillatory dependence $G\left(\Phi_{\mathrm{dc}}\right)$ is the standard mesoscopic interference effect similar to that in static electron interferometer. ${ }^{1}$ Another type of oscillating dependence, $G\left(A_{\mathrm{ac}}\right)$, arises from the Bessel function in Eq. (6). The dependence of conductance upon the ac power is shown in Fig. 2. The effect is, in fact, a classical interference between two light field amplitudes producing oscillating electron currents of the same frequency and coherent phase. Such oscillation require low enough temperature at which phase-breaking length of electron scattering $l_{\varphi}$ exceeds the circumference of the ring. Typically, $l_{\varphi}$ is of the order of inelastic (electron-electron or electron-phonon) scattering length. For the loop size of the order of $1 \mu \mathrm{m}$, the requirement $l_{\varphi}>L$ is valid for temperatures $T$ below $1 \mathrm{~K}$.

Another type of the amplitude damping at increasing temperature may be related to mismatch between the electron level spacing $\Delta \varepsilon$ and $T^{3,4}$ The latter effect is however not intrinsic to all kinds of electron interference. In the persistent-current type interference effects, ${ }^{3}$ vanishing of the oscillation amplitude at $T \gg \Delta \varepsilon$ arises as a result of averaging on the electron states. In the case of $\alpha$-dependent scattering as in Eq. (9), the average value of current for different states proves to be nonzero and thus does not remove the oscillating component of conductance. This means that, in principle, the effect of conductance oscillations can persist to temperatures and loop sizes larger than those in static interference experiments.

Another feature of quantum interference which we have not considered here may occur if the loop has unequal lengths of the upper and lower chains, e.g., as was shown in Refs. 14 and 15, the quantum flux periodicity may change from single to double one $(h c / l$ to $h c / 2 l)$.

We now turn to quantitative estimation of the effects under consideration. Let us put $L=1 \mu \mathrm{m}$. It follows from Eq. (10) that the magnitude of the field for which the dependence of $G$ on $\Phi_{\text {ac }}$ becomes important is of the order of $H_{\mathrm{ac}} \sim 10^{-7} \mathrm{~T}$ which corresponds to the oscillating power in the fiber $P \sim 10^{-3} w$. Estimated in a different way as a minimum number of optical photons transmitted through the ring which gives a further change in the phase oscillation of the order of $2 \pi$, the field should contain $N_{\omega} \sim 1 / \alpha$ photons where $\alpha$ is the fine structure constant $e^{2} / \hbar c$. Such change can be expected in the case of optical soliton propagating through the fiber. ${ }^{13}$

It should be stressed that the use of a nonuniform ring is very important for observation of the effects. Precisely, the above estimation of the critical value of $H_{\mathrm{ac}}$ crucially depends on the assumption made, that the ac power concentrates near some points in the ring because of its unhomogeneity. For a uniform ring the magnetic field corresponding to the effect of the order of $\Phi_{\mathrm{ac}} / N \Phi_{0}$ on the phase shift of the hopping amplitude between the nearest sites. In this case, the critical amplitude of the magnetic field has to be much higher $\left(H_{\mathrm{ac}} \sim 10^{-4} \mathrm{~T}\right)$, which corresponds to the oscillating power in the fiber of the order of $P \sim 10^{3} w$.

${ }^{1}$ S. Washburn, in Mesoscopic Phenomena in Solids, edited by B. L. Altshuler, P. A. Lee, and R. A. Webb (North-Holland, Amsterdam, 1991).

${ }^{2}$ Y. Aharonov and D. Bohm, Phys. Rev. 115, 485 (1959).

${ }^{3}$ I. O. Kulik, JETP Lett. 11, 275 (1970).

${ }^{4}$ M. Buttiker, Y. Imry, and R. Landauer, Phys. Lett. A 96, 365 (1983).

${ }^{5}$ Y. Gefen, Y. Imry, and M. Ya. Azbel, Phys. Rev. Lett. 52, 129 (1984).

${ }^{6}$ V. Chandrasekhar, R. A. Webb, M. J. Brady, M. B. Ketchen, W. J. Gallagher, and A. Kleinsasser, Phys. Rev. Lett. 67, 3578 (1991).

${ }^{7}$ D. Mally, C. Chapelier, and A. Benoit, Phys. Rev. Lett. 70, 2020 (1993).

${ }^{8}$ I. E. Aronov, E. N. Bogachek, and I. V. Krive, Phys. Lett. A 164, 331 (1992)

${ }^{9}$ I. E. Aronov, A. Grincwajg, M. Jonson, R. I. Shekhter, and E. N. Bogachek, Solid State Commun. 91, 75 (1994).

${ }^{10}$ L. D. Landau and E. M. Lifshitz, Mechanics (Pergamon, Oxford, 1976).

${ }^{11}$ R. Landauer, Philos. Mag. 21, 863 (1970).

${ }^{12}$ Y. Imry, in Directions in Condensed Matter Physics, edited by G. Grinstein and E. Mazenko (World Scientific, Singapore, 1986).

${ }^{13}$ G. Agrawal, Nonlinear Fiber Optics (Academic, Boston, 1989).

${ }^{14}$ C. H. Wu and G. Mahler, Phys. Rev. B 43, 5012 (1991).

${ }^{15}$ J. L. D'Amato, H. M. Pastawski, and J. F. Weisz, Phys. Rev. B 39, 3554 (1989). 